\title{
Conhecimento do enfermeiro nefrologista para atuar com o paciente em terapia renal substitutiva: hemodiálise
}

\author{
knowledge of nephrologist nurses to work with patients \\ in kidney replacement therapy: hemodialysis
}

Kleberson Tavares Pereira Dayane dos Santos Vieira ${ }^{1}$ Ana Fátima Souza Melo de ANDRADE $^{1}$

Taciana Silveira Passos ${ }^{\mathrm{I}}$

${ }^{\mathrm{I} C e n t r o}$ Universitário Estácio de Sergipe, Aracaju/SE - Brasil

IIUniversidade Tiradentes (Unit), Aracaju/SE - Brasil
Resumo A hemodiálise é o método mais utilizado atualmente pelos portadores da doença renal crônica. As medidas tomadas para o tratamento são sempre rigorosas e com graus de assimilação e adesão ao tratamento diversificado. Dessa forma, este estudo teve como objetivo identificar o nível de conhecimento da equipe de enfermagem em relação aos pacientes submetidos ao tratamento da hemodialise. Estudo descritivo, quantitativo, realizado por meio de um questionário estruturado aplicado aos profissionais de enfermagem do serviço de nefrologia em um hospital referência de Sergipe. Utilizou-se a estatística descritiva e o teste de Pearson para análise dos dados. 96,15\% da equipe de enfermagem conhece os tipos de tratamentos oferecidos ao paciente com Insuficiência Renal Crônica (IRC). 50\% relataram não conhecer a portaria 389 de 2014, destes 57,69\% não sabem informar quais são os parâmetros utilizados para diagnosticar um paciente com IRC. Entretanto, 76,92\% informaram ser necessário ter competência para atuar na nefrologia. Os que apresentaram maior conhecimento referente às competências para atuação na nefrologia foram os que participaram de capacitações $(26,03 \%)$ e os que conheciam a portaria $389(23,1 \%)$. Evidencia-se que há uma fragilidade no conhecimento da equipe de enfermagem e que se faz necessário a busca por novas capacitações na área, para a oferta de uma assistência qualificada e livre de eventos adversos.

Palavras-chave: Diálise renal; Cuidados de enfermagem; UniDADE DE HEMODIALISE.

Abstract Hemodialysis is currently the most used method by patients with chronic kidney disease. The measures taken for the treatment are always rigorous and with varying degrees of assimilation and adherence to the treatment. Thus, this study aimed to identify the level of knowledge of the nursing staff in relation to patients undergoing hemodialysis treatment. Descriptive, quantitative study, carried out through a structured questionnaire applied to nursing professionals in the nephrology service in a reference hospital in Sergipe. Descriptive statistics and the Pearson test were used for data analysis. $96.15 \%$ of the nursing staff know the types of treatments offered to patients with Chronic Kidney Failure (CRF). 50\% reported not knowing ordinance 389 of 2014, of which $57.69 \%$ are unable to inform which parameters are used to diagnose a 
patient with CRF. However, 76.92\% reported that it is necessary to have competence to work in nephrology. Those who had greater knowledge regarding skills for working in nephrology were those who participated in training (26.03\%) and those who knew the ordinance 389 (23.1\%). It is evident that there is a weakness in the knowledge of the nursing team and that it is necessary to search for new training in the area, in order to offer qualified assistance free of adverse events.

Keywords: Renal dialysis. Nursing Care. Hemodialysis unit.

\section{INTRODUÇÃo}

A insuficiência renal crônica é uma síndrome caracterizada pelo comprometimento dos rins ao executarem suas importantes funções. A disfunção nos rins promove diversas complicações no sistema humano, levando ao comprometimento do paciente ao quadro de renal crônico, tendo como alternativa de tratamento a diálise. ${ }^{1}$

$\mathrm{Na}$ atenção a pacientes renais, a realização do tratamento dialítico tem aumentado nas últimas décadas. Segundo a Sociedade Brasileira de Nefrologia, o número de pacientes em diálise passou de 42.695 em 2000 para 112.682 no ano de $2015 .^{2}$

A doença renal crônica (DRC) é caracterizada pela perda da funcionabilidade dos rins, classificando-se pela perca da taxa de filtração glomerular. Nos dias atuais a doença vem crescendo e se desenvolvendo de modo rápido acometendo grande parte da população, a qual tem como opção de tratamento a hemodiálise, diálise peritoneal ou o transplante renal. ${ }^{3}$

Os cuidados de enfermagem na nefrologia exigem algumas rotinas administrativas e orientações especiais para o paciente e sua família. O hemodialítico necessita de cuidados mais específicos, voltados para a assistência do enfermeiro que é o responsável por capacitar essa família. ${ }^{4}$
O profissional deve realizar uma assistência segura, evitando a ocorrência de infecções, atentando-se na manipulação dos dispositivos invasivos. Há precaução em evitar a ocorrência de eventos adversos, pois esses pacientes têm maior vulnerabilidade a incidentes como perca dos cateteres de hemodiálise, obstrução da via, perca da fistula e infecções na corrente sanguínea. ${ }^{5}$

$\mathrm{O}$ processo de enfermagem realizado de forma coerente e clara permite criar um diagnóstico mais completo, voltado para a assistência direcionado às queixas atuais do paciente, viabilizando a realização de intervenções e implementações afim da melhoria na qualidade de vida e de saúde do hemodialítico. ${ }^{6}$

Dessa forma, o estudo revela-se de suma importância para a construção de estratégias que assegurem uma melhor abordagem e manejo dos paciente renais crônicos. Por outro viés, atualmente é presente uma lacuna de conhecimentos e capacitações dos profissionais de enfermagem quanto ao manejo com pacientes renais. Objetivou-se identificar o nível de conhecimento da equipe de enfermagem em relação aos pacientes submetidos ao tratamento da hemodiálise.

\section{MATERIAL E MÉtodos}

\section{1 Delineamento e população do estudo}

Trata-se de um estudo descritivo com abordagem quantitativa com ênfase na atua- 
ção da equipe de enfermagem frente ao cuidado prestado ao paciente em tratamento hemodialítico em um hospital referência de Aracaju/SE, o qual ocorreu no período de agosto a outubro de 2019.

Segundo dados da Coordenação de Enfermagem do Hospital do Rim de Sergipe, a instituição hospitalar contém um dimensionamento de 30 profissionais de saúde. $\mathrm{O}$ estudo apresenta uma população de 05 enfermeiros e 25 técnicos de enfermagem distribuídos no setor de serviço de nefrologia.

\section{2 Amostra do estudo, critérios de inclusão e exclusão}

Para a seleção dos sujeitos da pesquisa foram levados em consideração os seguintes critérios: enfermeiros e profissionais da equipe de enfermagem que prestam $o$ atendimento a pacientes renais crônicos, no serviço de nefrologia do Hospital do Rim; enfermeiros e profissionais da equipe de enfermagem atuantes e em exercício com vínculo de pelo menos seis meses; foram excluídos 04 profissionais de enfermagem, pois eles apresentaram vínculo de trabalho inferior a seis meses na instituição de saúde, ou estavam de licença/férias no período da coleta e/ou recusou participar da pesquisa. Foram selecionados e entrevistados 26 profissionais de enfermagem do serviço de nefrologia.

\section{3 Instrumento e procedimento de coleta de dados}

Para caracterizar o perfil dos profissionais entrevistados, verificar as atividades e qualificar o atendimento prestado aos pacientes hemodialíticos, e para verificar o conhecimento dos profissionais de enfermagem do serviço de nefrologia, foi aplica- do um questionário estruturado e validado ${ }^{7}$ com questões fechadas com os horários previamente agendados com os participantes, os quais responderam verbalmente e o pesquisador marcou as respostas correspondentes no instrumento, após assinatura do termo de consentimento livre esclarecido.

Como variáveis dependentes teve-se o domínio dos profissionais de enfermagem para identificar os principais problemas apresentados pelos pacientes renais crônicos durante a hemodiálise; e as principais atividades executadas pelos profissionais de enfermagem para a prevenção e controle dos eventos adversos.

\section{4 Análise de dados}

Os dados foram processados no Programa Statistical Package for the Social Sciences (SPSS), versão 15.0, e apresentados por meio de estatísticas descritivas. Para caracterização dos profissionais, foi utilizada análise univariada por meio de distribuições de frequências em número absoluto e porcentagem. As questões foram classificadas em variáveis dicotómicas (corretas/adequadas e incorretas/inadequadas). Eram consideradas corretas/adequadas aquelas que estavam de acordo com as competências do enfermeiro especialista em nefrologia. ${ }^{7}$ As questões que tinham mais de uma alternativa correta só foram consideradas como adequadas quando o indivíduo respondia todas as alternativas corretas possíveis e as questões que não foram respondidas foram categorizadas como inadequadas.

Posteriormente, o teste qui-quadrado de Pearson foi utilizado para análise da distribuição das proporções de erros e acertos das questões com as características dos pro- 
fissionais. Para evitar resultados enviesados, foram excluídas aquelas variáveis que mesmo dicotomizadas apresentaram alternativas com frequência menor que cinco. $\mathrm{O}$ nível de significância estatística utilizado foi de $5 \%$ (p menor ou igual a 0,05 ).

\section{5 Princípios éticos}

Foram observados os aspectos éticos que regem pesquisas com seres humanos, conforme Resolução 466/12 do Conselho Nacional de Saúde. O estudo foi aprovado pelo Comitê de Ética em Pesquisa do Centro Universitário Estácio de Sergipe (CAAE de $n^{\circ}$ 18637519.0.0000.8079).

\section{Resultados e discussão}

O estudo permitiu identificar o nível de conhecimento da equipe de enfermagem em relação aos pacientes submetidos ao tratamento da hemodialise, quase a totalidade dos entrevistados afirmaram ter recebido capacitação para atuar na área de nefrologia e mais da metade deles referiram ter realizado capacitação $<1$ ano. A amostra foi composta predominantemente por um perfil de profissionais do sexo feminino, com faixa etária de 31 a 40 anos, sendo profissionais distribuídos entre enfermeiros e técnicos de enfermagem. Quanto ao tempo médio de atuação, a maioria atua entre 1 e 5 anos (Tabela 1).

Quanto à atuação dos profissionais de enfermagem e percepção sobre a logística operacional da unidade de nefrologia que exercem a profissão, observou-se que os pacientes com problemáticas são acompanhados por enfermeiros e por uma equipe multiprofissional em sua totalidade. As orientações em saúde ao cliente e a família são realizadas por $96,15 \%$ dos profissionais (Tabela 1). O que repercute positivamente para a potencialização do autocuidado ao paciente e da educação em saúde para paciente e família.

Em requisito de informações relevantes a serem passadas para o paciente e família, $73,08 \%$ informaram que é privativo do enfermeiro e $84,62 \%$ relataram que a estrutura da sala está adequada para prestação do serviço, seguindo o protocolo de segurança do paciente. Todos os entrevistados afirmaram a importância do domínio e cuidado com a manipulação do cateter. Evidenciou-se ser privativa do enfermeiro a supervisão dos procedimentos das máquinas no momento da desinfecção (Tabela 1). Ressaltando a minimização de eventos adversos relacionados à infecção e à garantia da segurança do paciente.

Tabela 1. Perfil profissiográfico e percepção sobre a logística operacional na unidade de nefrologia, Aracaju, 2019.

\begin{tabular}{lcc}
\hline & N & \% \\
\hline Faixa etária & & \\
\hline 25 a 30 anos & 10 & 38,46 \\
\hline 31 a 40 anos & 12 & 46,15 \\
\hline 41 a 50 anos & 3 & 11,54 \\
\hline Acima de 50 anos & 1 & 3,85 \\
\hline Sexo & & \\
\hline Masculino & 6 & 23,08 \\
\hline Feminino & 20 & 76,92 \\
\hline Atuação do profissional & & \\
\hline$\quad$ Enfermeiro & 5 & 19,23 \\
\hline $\begin{array}{l}\text { Técnico de } \\
\text { enfermagem }\end{array}$ & 20 & 76,92 \\
\hline
\end{tabular}




\begin{tabular}{|c|c|c|}
\hline $\begin{array}{l}\text { Auxiliar de } \\
\text { enfermagem }\end{array}$ & 1 & 3,85 \\
\hline \multicolumn{3}{|l|}{$\begin{array}{l}\text { Tempo de atuação na } \\
\text { nefrologia }\end{array}$} \\
\hline 1 a 5 & 15 & 57,69 \\
\hline 6 a 10 & 10 & 38,46 \\
\hline$>10$ & 1 & 3,85 \\
\hline \multicolumn{3}{|c|}{$\begin{array}{l}\text { Capacitação para atuar no setor de } \\
\text { nefrologia }\end{array}$} \\
\hline Sim & 19 & 14,8 \\
\hline Não & 7 & 26,92 \\
\hline 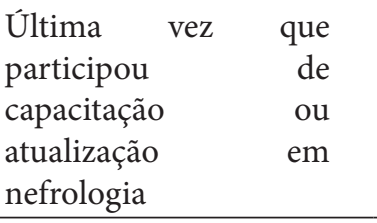 & & \\
\hline$<1$ ano & 18 & 69,23 \\
\hline$>2$ anos & 1 & 3,85 \\
\hline Não se aplica & 7 & 26,92 \\
\hline $\begin{array}{l}\text { Problemas dos } \\
\text { pacientes são avaliados } \\
\text { e registrados por um } \\
\text { enfermeiro }\end{array}$ & $\mathbf{N}$ & \\
\hline Sim & 26 & 100,0 \\
\hline \multicolumn{3}{|l|}{$\begin{array}{l}\text { Paciente é } \\
\text { acompanhado por uma } \\
\text { equipe multiprofissional }\end{array}$} \\
\hline \multicolumn{3}{|l|}{ Sim } \\
\hline $\begin{array}{l}\text { Sala de hemodiálise com } \\
\text { estrutura adequada para } \\
\text { a prestação do serviço }\end{array}$ & 26 & 100,0 \\
\hline Sim & 22 & 84,62 \\
\hline \multicolumn{3}{|l|}{ Não } \\
\hline $\begin{array}{l}\text { Atuação de acordo } \\
\text { com o Protocolo de } \\
\text { Segurança do Paciente }\end{array}$ & 4 & 15,38 \\
\hline Sim & 26 & 100,0 \\
\hline $\begin{array}{l}\text { Realiza orientação em } \\
\text { saúde ao cliente e a } \\
\text { família }\end{array}$ & & \\
\hline
\end{tabular}

\begin{tabular}{lcc}
\hline Sim & 25 & 96,15 \\
\hline Não & 1 & 3,85 \\
\hline $\begin{array}{l}\text { Percepção de } \\
\text { importância do } \\
\text { domínio dos cuidados } \\
\text { e da manipulação dos } \\
\text { cateteres e fístulas }\end{array}$ & \\
\hline Sim & & \\
\hline
\end{tabular}

Toda informação e ensino relevantes dados aos pacientes/família são da responsabilidade do enfermeiro

\begin{tabular}{lcc}
\hline Sim & 19 & 73,08 \\
\hline Não & 6 & 23,08 \\
\hline Não sabe informar & 1 & 3,85 \\
\hline
\end{tabular}

Realiza consulta de enfermagem (enfermeiro)

\begin{tabular}{lcc}
\hline Sim & 5 & 19,23 \\
\hline Não se aplica & 21 & 80,77 \\
\hline
\end{tabular}

\section{Realiza orientação}

e supervisão dos procedimentos de desinfecção de equipamentos e de reprocessamento dos dialisadores (enfermeiro)

\begin{tabular}{lcc}
\hline Sim & 5 & 19,23 \\
\hline Não se aplica & 21 & 80,77 \\
\hline
\end{tabular}

Outrora, no estudo de Cesarino et al. ${ }^{8}$ penas 50,6\% afirmaram que os problemas los pacientes são avaliados e registrados or uma enfermeira. De acordo com autoes especialistas em nefrologia, ${ }^{9-10}$ todo paiente deve ter o tratamento supervisionado or uma equipe multiprofissional e espeializada, tendo como objetivo condutas de lesenvolvimentos diante do agravamento 
ou para retardar a evolução da doença. Os conhecimentos sobre a segurança do paciente devem ser frequentes e atuais para que, assim, a equipe de enfermagem esteja capacitada para evitar riscos à saúde do paciente garantindo uma melhor assistência e proporcionando uma melhor qualidade de vida para o paciente em tratamento.

Silva et al. ${ }^{11}$ ressaltam que o profissional de enfermagem deve buscar ações em educação em saúde conforme a necessidade do paciente e seu familiar, essa estratégia visa orientar e acolher melhor cada indivíduo para que, assim, adequem-se ao novo estilo de vida e melhor adesão ao tratamento.

Dentre as principais complicações apresentadas pelos pacientes no momento da hemodiálise, a obstrução do cateter foi a mais referida $(80,77 \%)$. Em relação as orientações no momento da admissão do paciente, $73,08 \%$ informaram esclarecer as dúvidas sobre a doença e terapia substitutiva, porém a depender do comportamento do paciente passam a responsabilidade para a família em explicar ao paciente sobre a doença (92,31\%), conduta essa divergente do que é preconizado pela Portaria 389 de 2014; 57,69\% afirmaram ter como competência a assistência, apenas ligar o paciente na máquina e mantê-lo sem intercorrência. Quanto a percepção de intercorrência no momento do tratamento foi relatada pela maioria $(84,62 \%)$ que a atenção e a agilidade são os métodos mais utilizados em situações de risco (Tabela 2). Ressaltando a eficiência e eficácia da equipe no atendimento.

Em estudo realizado com 31 pacientes em sessões de hemodiálise em uma unidade de terapia intensiva de um hospital público no ano de 2018 foram destacados como principais ocorrências a hipotensão, hipotermia, hipoglicemia, coagulação do siste- ma, arritmias cardíacas, sangramento e falta de fluxo no acesso vascular. ${ }^{12}$ Em uma pesquisa no Centro Hospitalar de Lisboa Norte, a perda do dispositivo foi a complicação mais frequente com acessos vasculares. ${ }^{13}$

Diante da atenção e agilidade na percepção de situações de risco no processo dialítico, Andrade et al. ${ }^{14}$ evidenciaram que o enfermeiro tinha o discernimento em tomar decisões quanto à intercorrência que o paciente apresentasse.

Segundo Oliveira e Soares, ${ }^{15}$ a equipe é a responsável por acolher o paciente e deve ter competência assistencial, explicando todos os tipos de tratamentos possíveis para que ele possa escolher o melhor método a aderir, estando ciente das possíveis complicações e intercorrências que estará susceptível.

Quase a totalidade da equipe de enfermagem conhece os tipos de tratamentos oferecidos ao paciente com IRC (96,15\%). Dentre os tipos de tratamento foram citados hemodiálise, diálise peritoneal e transplante renal por $76,92 \%$ dos profissionais. $50 \%$ relataram não conhecer a portaria 389 de 2014, destes 57,69\% não sabem informar quais são os parâmetros utilizados para diagnosticar um paciente com insuficiência renal crônica. 76,92\% informaram ser necessário ter competência para atuar na nefrologia, onde o conhecimento prático e o embasamento teórico são de extrema importância. Entretanto os dados demonstram a possibilidade de risco ao evento adverso na prestação do cuidado visto que a maioria dos entrevistados não conhecem a portaria que regula o serviço de nefrologia e não identificam os parâmetros para diagnóstico. Quanto a competência profissional, 11,54\% dos enfermeiros relataram exercer funções assistenciais, administrativas e educativas (Tabela 2). 
Tabela 2. Conhecimento sobre o perfil de atendimento e atuação profissional na unidade de nefrologia, Aracaju, 2019.

Legenda: RC: Renal Crônico; ICR: Insuficiência Renal Crônica.

\begin{tabular}{|c|c|c|c|c|}
\hline & \multicolumn{2}{|c|}{ Sim } & \multicolumn{2}{|c|}{ Não } \\
\hline & $\mathbf{N}$ & $\%$ & $\mathbf{N}$ & $\%$ \\
\hline \multicolumn{5}{|l|}{$\begin{array}{l}\text { Principais complicações apresentadas pelo RC na sessão de } \\
\text { hemodiálise }\end{array}$} \\
\hline Hipotensão & 18 & 69,23 & 8 & 30,77 \\
\hline Calafrios & 16 & 61,54 & 10 & 38,46 \\
\hline Obstrução de cateter & 16 & 61,54 & 10 & 38,46 \\
\hline Câimbras & 15 & 57,69 & 11 & 42,31 \\
\hline \multicolumn{5}{|l|}{ Maiores complicações com acesso vasculares da hemodiálise } \\
\hline Infecções na corrente sanguínea & 15 & 57,69 & 11 & 42,31 \\
\hline Obstrução de cateter & 21 & 80,77 & 5 & 19,23 \\
\hline Perca do dispositivo & 5 & 19,23 & 21 & 80,77 \\
\hline \multicolumn{5}{|l|}{ Orientações quando o paciente é admitido } \\
\hline $\begin{array}{c}\text { Esclarecer dúvidas sobre doença renal crônica e terapia renal } \\
\text { substitutiva, além de reduzir o medo da máquina de hemodiálise }\end{array}$ & 19 & 73,08 & 7 & 26,92 \\
\hline $\begin{array}{l}\text { Passar toda a responsabilidade do tratamento a depender do } \\
\text { comportamento do cliente }\end{array}$ & 2 & 7,69 & 24 & 92,31 \\
\hline $\begin{array}{l}\text { Reduzir o medo da máquina de hemodiálise e responsabilizar o } \\
\text { familiar a explicar ao paciente sobre a doença renal crônica }\end{array}$ & 2 & 7,69 & 24 & 92,31 \\
\hline Não sabe informar & 5 & 19,23 & 20 & 76,92 \\
\hline \multicolumn{5}{|l|}{ Competência da assistência } \\
\hline $\begin{array}{l}\text { Identificação juntamente com o médico, indivíduo e família sobre o } \\
\text { melhor método dialítico para aquele cliente. }\end{array}$ & 7 & 26,92 & 19 & 73,08 \\
\hline Interpretação de exames laboratoriais & 11 & 42,31 & 15 & 57,69 \\
\hline Ligar o paciente a máquina e mantê-lo sem risco para intercorrências & 15 & 57,69 & 11 & 42,31 \\
\hline $\begin{array}{l}\text { Admitir o paciente e encaminhá-lo para a consulta médica para que } \\
\text { este defina o tratamento a ser implementado }\end{array}$ & 5 & 19,23 & 21 & 80,77 \\
\hline Não sabe informar & 3 & 11,54 & 23 & 88,46 \\
\hline \multicolumn{5}{|l|}{ Como perceber intercorrências ao longo do procedimento dialítico } \\
\hline Experiência prática & 21 & 80,77 & 5 & 19,23 \\
\hline Conhecimento científico & 19 & 73,08 & 7 & 26,92 \\
\hline Atenção e agilidade em perceber situações de risco & 22 & 84,62 & 4 & 15,38 \\
\hline $\begin{array}{l}\text { Conhece os tratamentos que podem ser oferecidos ao paciente com } \\
\text { IRC }\end{array}$ & 25 & 96,15 & 1 & 3,85 \\
\hline
\end{tabular}




\section{Tipos de tratamento para a IRC}

\begin{tabular}{lcccc}
\hline Diálise peritoneal & 22 & 84,62 & 4 & 15,38 \\
\hline Hemodiálise & 25 & 96,15 & 1 & 3,85 \\
\hline Transplante renal & 20 & 76,92 & 6 & 23,08 \\
\hline Litotripsia & 3 & 11,54 & 3 & 11,54 \\
\hline Medicamentoso & 2 & 7,69 & 2 & 7,69 \\
\hline Não sabe informar & 1 & 3,85 & 25 & 96,15 \\
\hline
\end{tabular}

Conhece a Portaria 389 de 2014

\begin{tabular}{lcccc}
\hline Competências necessárias para atuar na nefrologia & 13 & 50,00 & 13 & 50,00 \\
\hline O conhecimento prático aprofundado e o pensamento crítico & 24 & 92,31 & 2 & 7,69 \\
\hline O direcionamento do cuidado e o embasamento teórico nas ações & 22 & 84,62 & 4 & 15,38 \\
\hline
\end{tabular}

\section{Competências profissionais (enfermeiro)}

\begin{tabular}{lrrrr}
\hline Assistenciais & 3 & 60,00 & 2 & 40,00 \\
\hline Administrativas & 3 & 60,00 & 2 & 40,00 \\
\hline Educativas & 3 & 60,00 & 2 & 40,00 \\
\hline Pesquisas & 2 & 40,00 & 3 & 60,00 \\
\hline Não se aplica & 21 & 80,77 & 5 & 19,23 \\
\hline
\end{tabular}

Em um estudo realizado em Recife com profissionais de saúde no serviço de nefrologia a capacitação ainda tem um percentual baixo, ao qual não possuem especialização para atuar na área.16 De acordo com a análise dos dados da portaria 389 de 2014, o artigo 31 exige competência dos enfermeiros com capacitação e especialidade para atuar na nefrologia. ${ }^{17}$

Observou-se quanto a percepção dos profissionais de enfermagem que $76,92 \%$ estão satisfeitos com os cuidados prestados. Em relação às interferências do tratamento na vida do paciente, foram citados os fatores sexual, social e psicológico, não sendo considerados os fatores financeiro e a alimentação como fator interferente. $73,08 \%$ relataram que os pacientes apresentam resistência em aceitar a doença e aderir a mudança de hábitos (Tabela 3).

Semelhante a Noleto et al., ${ }^{18}$ em seu estudo, 73,08\% afirmaram que as dificuldades ao tratamento estão associadas à resistência para aceitação da doença, como mudanças de hábitos e relacionamento com os familiares. $\mathrm{O}$ que pode ser considerado como um entrave para progressão ao tratamento. Diante disso, os profissionais de saúde declararam que utilizam múltiplas estratégias para auxiliar no enfrentamento psicológico, como a religião e apoio da família. ${ }^{19}$

Com base nas perguntas direcionadas à equipe de enfermagem relacionadas ao conhecimento frente à assistência ao paciente, percebeu-se na figura 1 que ainda existe fragilidade pela equipe de enfermagem na prestação desse cuidado. Foi aplicado um questionário estruturado no qual as pergun- 
Tabela 3. Percepção dos profissionais de enfermagem sobre satisfação, interferência do tratamento na vida e enfretamento do paciente à doença renal crônica.

\begin{tabular}{lcc}
\hline Percepção de satisfação dos pacientes com os cuidados prestados & N & $\%$ \\
\hline Sim & 20 & 76,92 \\
Não & 5 & 19,23 \\
Não sabe informar & 1 & 3,85 \\
Percepção de interferência do tratamento na vida do paciente com IRC & & \\
Financeira & 10 & 38,46 \\
$\quad$ Sim & 16 & 61,54 \\
Não & & \\
Sexual & 23 & 88,46 \\
Sim & 3 & 11,54 \\
Não & & \\
Social & 21 & 80,77 \\
Sim & 5 & 19,23 \\
Não & & \\
Alimentação & 11 & 42,31 \\
Sim & 15 & 57,69 \\
Não & & \\
Psicológico & 25 & 96,15 \\
Sim & 1 & 3,85 \\
Não & & \\
Percepção de enfrentamento do paciente ao ser diagnosticado com a doença renal \\
crônica \\
Os pacientes sempre contam com o suporte da família \\
Os pacientes apresentam resistência em aceitação da doença e mudança de hábitos \\
Alguns pacientes contam com suporte da família, outros pensam de imediato em \\
desistir e outros apresentam resistência em aceitar a doença e mudança de hábitos & 19 & 33,08 \\
\hline
\end{tabular}

Legenda: IRC: Insuficiência Renal Crônica.

tas tinham como alternativas de respostas mais que uma opção, os resultados obtidos como incorretos/inadequados destacados na cor vermelha da figura foram daqueles que não conseguiram identificar todas as opções corretas, já os azuis referem-se ao correto/ adequado, e obtiveram um percentual maior.

Quanto às competências, os entrevistados responderam corretamente a forma de atuação no setor de nefrologia, em contraponto quando questionados sobre os parâmetros utilizados para diagnosticar o paciente com insuficiência renal crônica, $70 \%$ dos entrevistados apresentaram respostas erra- das. Apenas 50\% dos entrevistados relataram conhecer a Portaria no 389 de 2014 e quase a totalidade responderam não saber pontuar como pode ser identificado a ocorrência ao longo do procedimento dialítico, 70\% destes não souberam identificar as maiores complicações com o cateter vascular de hemodiálise. Diante disso, propõe-se a elaboração de estratégias, com oferta de capacitações contínuas assegurando a redução e inexistência dos eventos adversos.

Observa-se que $100 \%$ dos entrevistados afirmaram que os problemas dos pacientes são acompanhados e registrados pelo en- 
Competências necessárias para atuar na nefrologia

Parâmetros utilizados para o diagnóstico da IRC

Conhece a Portaria 389 de 2014

Tipos de tratamento para a IRC

Conhece os tipos de tratamentos que podem ser oferecidos ao paciente com IRC

Como é possivel perceber intercorrências ao longo do procedimento dialitico

Competência da assistência (enfermeiro)

Orientações quando o paciente é admitido

Maiores complicações com acesso vasculares da hemodiálise

Principais complic ações apresentadas pelo paciente renal crônico durante a sessão de hemodiálise

Realiza orientação e supervisão dos procedimentos de desinfecção de equipamentos e de...

Realiza consulta de enfermagem (enfermeiro)

Toda informação e ensino relevantes dados aos pacientes/familia são da responsabilidade do..

Percepção de importância do dominio dos cuidados e da manipulação dos cateteres e fistulas

Realiza orientação em saúde ao cliente e a família

Atuação de acordo com o Protocolo de Segurança do Paciente

Sala de hemodiálise com estrutura adequada para a prestação do serviço

Paciente é acompanhado por uma equipe mul tiprofissional

Problemas dos pacientes são avaliados e registrados por um enfermeiro
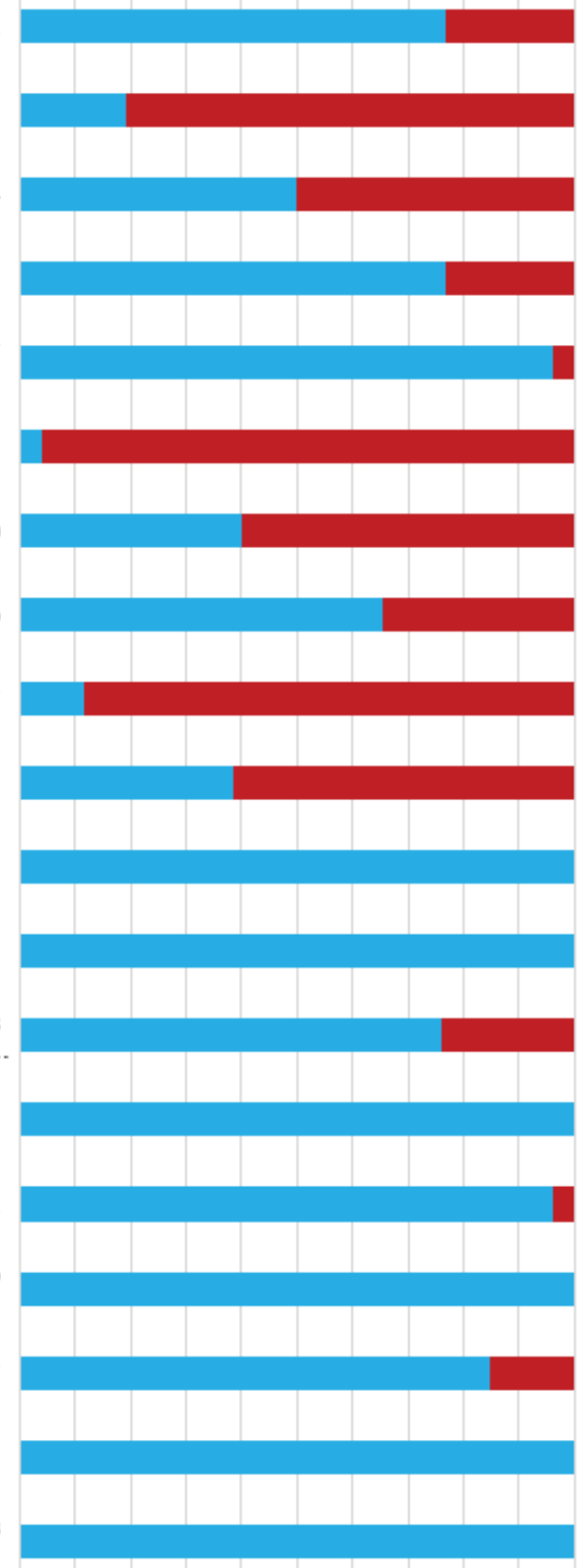

$0 \% 10 \% 20 \% 30 \% 40 \% 50 \% 60 \% 70 \% 80 \% 90 \% 100 \%$

m Correto/ adequado Incorreto/inadequado

Figura 1. Distribuição das respostas dos profissionais de enfermagem para cada item

relacionado à atuação e conhecimento frente à assistência em nefrologia.

Nota: Aqueles que não marcaram todas as alternativas possíveis correspondentes à questão, foram categorizados em incorretos/inadequados por não acertarem por completo. IRC - Insuficiência Renal Crônica 
fermeiro. Semelhante ao que Andrade et al. ${ }^{20}$ pontuaram, que os problemas ocorridos durante o procedimento de diálise são acompanhados e registrados pelo enfermeiro da equipe. $\mathrm{O}$ que reflete uma assistência de maior qualidade e segurança, por ser ofertada por uma profissional de maior capacidade técnica e conhecimento teórico e prático.

Já no estudo de Silva e Matos, ${ }^{21}$ as condutas de intervenção são realizadas e registradas rotineiramente por toda a equipe de enfermagem na realização de quaisquer tipos de procedimento, reforçando que é imprescindível manter a equipe capacitada e atualizada.

Ressalta-se que os entrevistados em sua totalidade afirmaram que os pacientes são acompanhados por uma equipe multiprofissional. $\mathrm{O}$ paciente e familiar recebem a assistência multiprofissional, sendo o enfermeiro responsável por orientá-los sobre as complicações, limitações, a importância de um apoio familiar/amigos ou até um acompanhamento psicológico. ${ }^{22}$

Ao associar as condutas e conhecimentos sobre atuação na nefrologia com a caracterização dos profissionais, pode-se observar uma maior proporção de acertos de indivíduos mais jovens, enfermeiros, menor tempo de atuação, aqueles que participaram de capacitação e conhecem a Portaria no 389 de 2014 (Tabela 4).

Sendo que, destaca-se três associações que foram estatisticamente significativas: os profissionais enfermeiros tiveram maior proporção de acertos que os técnicos de enfermagem na pergunta sobre os parâmetros utilizados para o diagnóstico da IRC $(\mathrm{p}=0,01)$; os que participaram de capacitação acertaram mais os tipos de tratamento para IRC

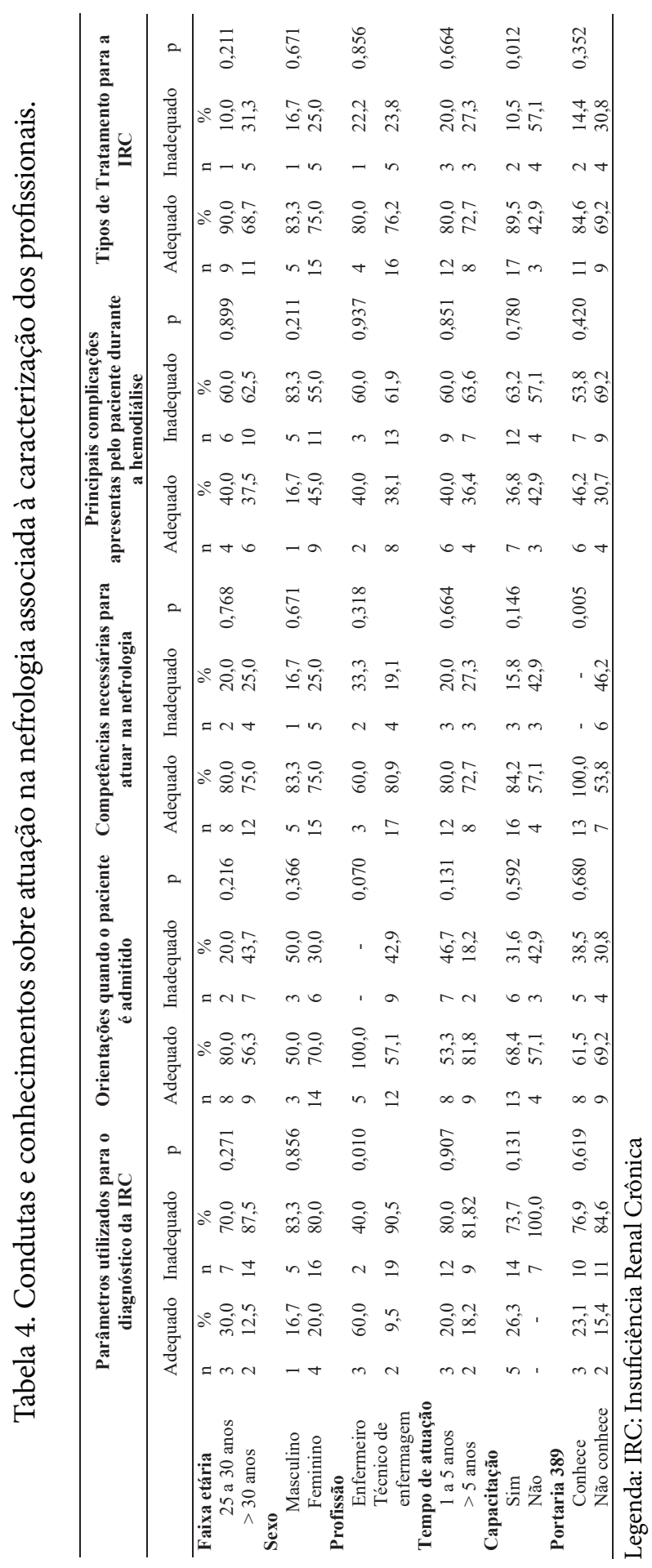


comparados àqueles que nunca participaram de capacitação $(\mathrm{p}=0,012)$. Os profissionais que conheciam a Portaria no 389 de 2014 tiveram maior frequência de acertos na questão sobre as competências necessárias para a atuação na nefrologia ( $\mathrm{p}=0,005)$.

Ressalta-se que a capacitação visa ampliar a qualificação e o aprimoramento dos profissionais de enfermagem, havendo uma necessidade na busca pelo conhecimento constante.7 A grande carência de conhecimento da equipe de enfermagem de nefrologia se depara com a escassez de busca e especializações potencializando uma condição vulnerável desses profissionais, impossibilitando a autonomia para intervir nos cuidados prestados para os pacientes.

\section{Conclusão}

Diante do que foi discutido, constata-se a importância do papel do enfermeiro como educador e facilitador da atenção ao cuidado, sendo exigidas habilidades especiais, assim como entendimento dos sentimentos que são expressos, no momento da sessão de hemodiálise. Além disso, enfatiza-se quanto à falta de capacitação na área, sendo inclusa como fator que dificulta os cuidados. Desse modo, observa-se que o campo da nefrologia é vasto para a atuação do profissional da enfermagem, no intuito de que a profissão procura conhecer as necessidades de seus pacientes para prestar uma assistência diferenciada, traçando estratégias que procurem minimizar não só os desconfortos físicos do tratamento, mas também os emocionais.

Percebe-se o baixo conhecimento dos entrevistados sobre a Portaria no 389 de 2014, principalmente no que se refere às competências necessárias para a atuação na nefrologia, $\mathrm{o}$ que ressaltam a importância da busca por novos conhecimentos, contribuindo para o bem-estar e segurança do paciente, consequentemente a melhoria da qualidade assistencial. Verifica-se a necessidade de mais estudos com amostragem maior para compreender a visão dos enfermeiros nefrologistas.

\section{REFERÊNCIAS}

1. Alves LD, Guedes CC, Costa BG. As ações do enfermeiro ao paciente renal crônico: reflexão da assistência no foco da integralidade. Rev. Pesqui. (Univ. Fed. Estado Rio J., Online). 2016:3907-21.

2. Aguiar LL, Guedes MV, Oliveira RM, Leitão IM, Pennafort VP, Barros AA. Enfermagem e metas internacionais de segurança: avaliação em hemodiálise. Cogitare enferm. 2017;22(3):e45609.

3. Ottaviani AC, Betoni LC, Pavarini SC, Gramani Say K, Zazzetta MS, Orlandi FD. Associação entre ansiedade e depressão e a qualidade de vida de pacientes renais crônicos em hemodiálise. Texto contexto - enferm. 2016;25(3).

4. Marques FR, Botelho MR, Marcon SS, Pupulim JS. Estratégias de coping utilizadas por familiares de indivíduos em tratamento hemodialítico. Texto contexto - enferm. 2014 Dec;23(4):915-24.

5. Silva GG, Nunes JT, Barboza IR, Barros TR, Souza ÂM, Davim RM, et al. Distúrbios renais em unidade de terapia intensiva. Revista de enfermagem. Rev. enferm. UFPE on line. 2017:4463-8.

6. Grassi MD, Dell'Acqua MC, Jensen R, Fontes CM, Guimarães HC. Diagnósticos, resultados e intervenções de enfermagem em pacientes com lesão renal aguda. Acta paul. enferm. 2017;30(5):538-45.

7. Oliveira NB, Costa FV, Assad LG. Competências do enfermeiro especialista em nefrologia. Rev. enferm. UERJ. 2015;23(3):375-80. 
8. Cesarino CB, Ribeiro RD, Lima IC, Bertolin DC, Ribeiro DF, Rodrigues AM. Avaliação do grau de satisfação de pacientes renais crônicos em hemodiálise. Acta paul. enferm. 2009;22(SPE1):51923.

9. Castro MC. Tratamento conservador de paciente com doença renal crônica que renuncia à diálise. J. bras. nefrol. 2019;41(1):95-102.

10. Rocha RD, Pinho DL. Segurança do paciente em hemodiálise. Rev. enferm. UFPE on line. 2018:3360-7.

11. Silva AD, Souza AT, Arenas VG, Barros LF. A ação do enfermeiro na prevenção de doença renais crônicas: uma revisão integrativa. SANARE. 2015;14(2):148-55.

12. Silva AF, Magalhães DM, Rocha PR, da Silva RF. Principais complicações apresentadas durante a hemodiálise em pacientes críticos e propostas de intervenções de enfermagem. R. Enferm. Cent. O. Min. 2018;8:2327.

13. Pereira M, Lopez N, Godinho I, Jorge S, Nogueira E, Neves F, et al. Acesso vascular life-saving na exaustão do capital vascular: experiência de um centro com cateteres intra-auriculares para hemodiálise. J. bras. nefrol. 2017;39(1):36-41.

14. Andrade BR, Barros FD, Lúcio HF, Campos JF, Silva RC. Atuação do enfermeiro intensivista no modelo colaborativo de hemodiálise contínua: nexos com a segurança do paciente. Rev. Esc. Enferm. USP. 2019;53.

15. de Oliveira AM, Soares E. A Comunicação como Importante Ferramenta nas Orientações em uma Unidade de Hemodiálise: um estudo reflexivo. Saúde transform. soc. 2014;5(3):118-23.

16. Ferraz RN, de Godoy Maciel C, Borba AK, da Silva Frazão I, França VV. Percepção dos profissionais de saúde sobre os fatores para a adesão ao tratamento hemodialítico. Rev. enferm. UERJ. 2017;25:15504.

17. Ministério da Saúde (BR). Portaria n 389, de 13 de março de 2014. Define os critérios para organização da linha de cuidado para pessoa com doença renal crônica e institui incentivo financeiro de custeio destinado ao cuidado ambulatorial pré-dialítico.

18. Noleto LC, Fonseca AC, Luz MH, Batista OM, Pereira AF. O papel dos profissionais de enfermagem no cuidado ao paciente em tratamento hemodialítico?: revisão integrativa. Rev. enferm. UFPE on line. 2015;9:1580-6.

19. Silva RA, Souza Neto VL, Oliveira GJ, Silva BC, Rocha CC, Holanda JR. Estratégias de enfrentamento utilizadas por pacientes renais crônicos em tratamento hemodialítico. Esc. Anna Nery Rev. Enferm. 2016 Mar;20(1):147-54.

20. Andrade BR, Barros FD, Lúcio HF, Campos JF, Silva RC. Experiência de enfermeiros no manejo da hemodiálise contínua e suas influências na segurança do paciente. Texto contexto - enferm. 2019;28.

21. Silva PE, de Mattos M. Complicações hemodialíticas na Unidade de Terapia Intensiva. Rev. enferm. UFPE on line. 2019:162-8.

22. Frazão CM, Delgado MF, Araújo MG, Silva FB, Sá JD, Lira AL. Cuidados de enfermagem ao paciente renal crônico em hemodiálise. Rev Rene (Online). 2014;15(4):701-9. 


\section{DADOS DOS AUTORES}

\section{Kleberson Tavares Pereira}

Graduado no Centro Universitário Estácio de Sergipe, Aracaju/SE - Brasil.

DAYANE dos Santos Vieira

Graduada no Centro Universitário Estácio de Sergipe, Aracaju/SE - Brasil.

\section{Ana Fátima Souza Melo de Andrade}

Mestra em saúde e ambiente. Orientadora. Docente do curso de enfermagem do Centro Universitário Estácio de Sergipe, Aracaju/SE - Brasil. anafatimamelo@hotmail.com

\section{Taciana Silveira Passos}

Doutoranda e Mestre em saúde e ambiente na Universidade Tiradentes (Unit), Aracaju/SE Brasil. tac

Submetido em: 5-4-2021

Aceito em: 26-10-2021 